\title{
The invasive Korean bush mosquito Aedes koreicus (Diptera: Culicidae) in Germany as of 2020
}

Nicolas Hohmeister ${ }^{1}$, Doreen Werner ${ }^{2}$ and Helge Kampen ${ }^{1 *}$

\begin{abstract}
Background: The Korean bush mosquito Aedes koreicus was recently reported to have established a population in western Germany (Wiesbaden) in 2016. The species is difficult to distinguish morphologically from its close relative, the invasive Japanese bush mosquito Ae. japonicus, which is already widely distributed in many parts of Germany, including the area colonised by Ae. koreicus. Genetic confirmation of morphologically identified "Ae. japonicus" collection material, however, had only been done exceptionally before the German Ae. koreicus population became known.

Methods: Dried archived "Ae. japonicus" specimens both from the municipality of Wiesbaden and from deliberately and randomly selected distribution sites all over Germany were re-examined morphologically and genetically for admixture by Ae. koreicus. Moreover, cemeteries in the greater Wiesbaden area were sampled in 2019 and 2020 to check for Ae. koreicus spread. Korean and Japanese bush mosquitoes submitted to the German citizen science mosquito monitoring scheme "Mueckenatlas" in 2019 and 2020 were also subjected to particularly thorough species identification. The ND4 DNA sequences generated in this study in the context of species identification were phylogenetically compared to respective GenBank entries of Ae. koreicus. As a by-product, several genetic markers were evaluated for their suitability to identify Ae. koreicus.
\end{abstract}

Results: Aedes koreicus specimens could be identified in mosquito collection material and submissions from Wiesbaden from 2015 onwards, suggesting establishment to have happened in the same year as Ae. japonicus establishment. Detections of Ae. koreicus from 2019 and 2020 in Wiesbaden indicate a negligible enlargement of the populated area as described for 2018. Two Ae. koreicus specimens were also submitted from the city of Munich, southern Germany, in 2019 but further specimens could not be identified during immediate local inspections. Comparison of ND4 sequences generated in this and other studies demonstrate a high degree of homology, suggesting that this DNA region is not informative enough for clarification of origins and relationships of Ae. koreicus populations. For genetic identification of Ae. koreicus, PCR primers used for classical CO1 barcoding were found to lead to mismatches and produce no or incorrect amplicons. Alternative CO1 primers or a validated ND4 marker should be used instead.

Conclusions: Aedes koreicus is probably introduced into Germany every now and then but rarely succeeds in becoming established. As with most European populations, the German population is characterised by a limited expansion tendency. Since Ae. koreicus is a potential vector, however, Asian bush mosquitoes found at new places should

*Correspondence: helge.kampen@fli.de

1 Friedrich-Loeffler-Institut, Federal Research Institute for Animal Health, Insel Riems, Greifswald, Germany

Full list of author information is available at the end of the article original author(s) and the source, provide a link to the Creative Commons licence, and indicate if changes were made. The images or other third party material in this article are included in the article's Creative Commons licence, unless indicated otherwise in a credit line to the material. If material is not included in the article's Creative Commons licence and your intended use is not permitted by statutory regulation or exceeds the permitted use, you will need to obtain permission directly from the copyright holder. To view a copy of this licence, visit http://creativecommons.org/licenses/by/4.0/. The Creative Commons Public Domain Dedication waiver (http://creativeco mmons.org/publicdomain/zero/1.0/) applies to the data made available in this article, unless otherwise stated in a credit line to the data. 
be examined quite carefully and known distribution areas of Ae. japonicus regularly checked for the presence of Ae. koreicus.

Keywords: Aedes japonicus, Cemetery, Distribution, Genetic identification, Hulecoeteomyia koreica, Invasive mosquito species, Germany, Mueckenatlas

\section{Graphical Abstract}

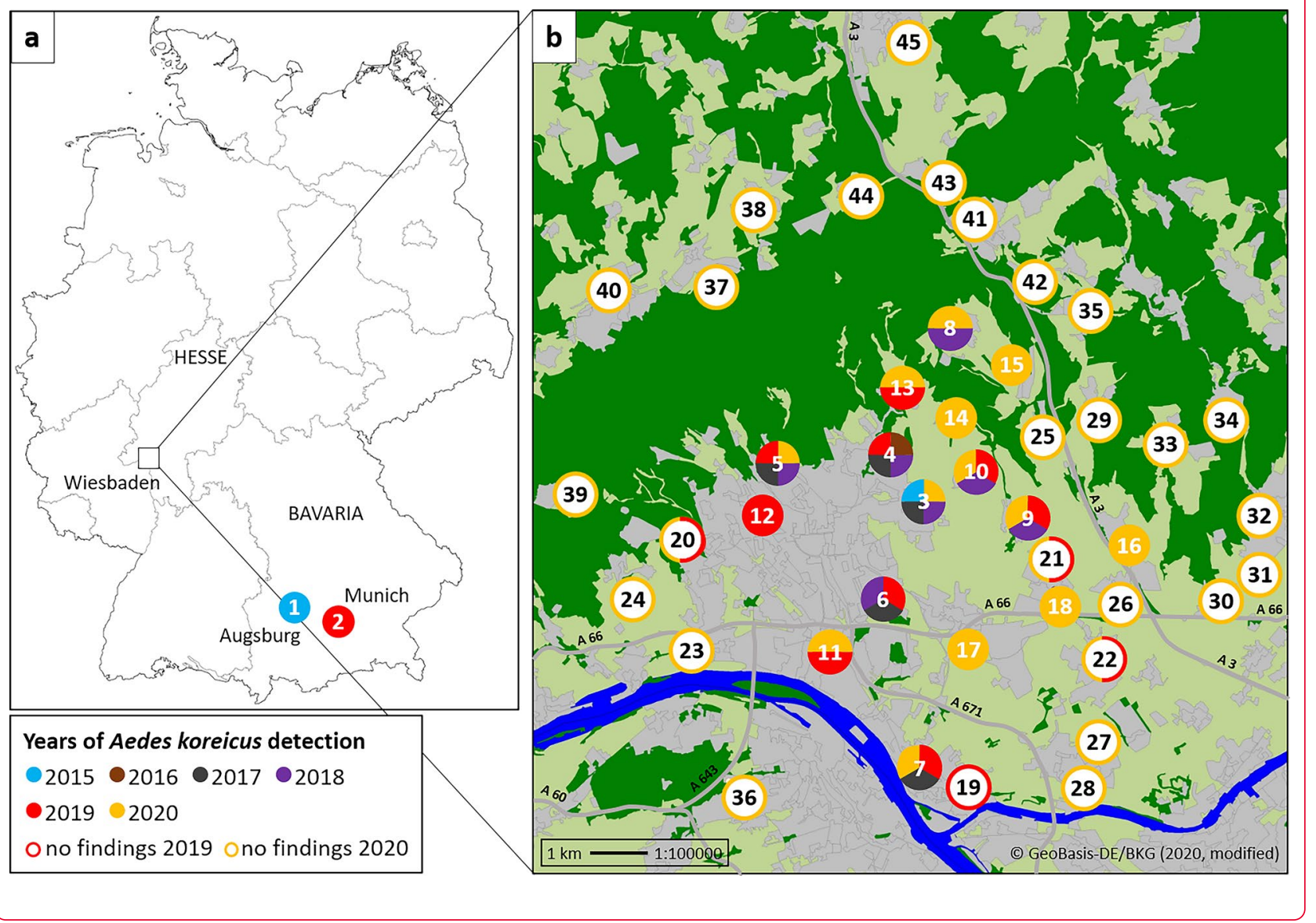

\section{Background}

The Korean bush mosquito, Aedes (Hulecoeteomyia) koreicus (Edwards, 1917), has its native distribution area in Japan, Korea, northern China and southern Russia [1, 2]. Following Ae albopictus, Ae aegypti and Ae. japonicus [3], it became invasive to Europe in 2008 when the first specimens were reported from Belgium [4]. In 2011, the species emerged in Italy, in 2013 in Russia (eastern Black Sea coast), Switzerland and Slovenia, in 2015 in Germany, in 2016 in Hungary and in 2018 in Austria [5-11]. In contrast to Italy, where the species spread after establishment [12, 13], no significant dispersal has been reported from other colonised areas, while the detection of a single specimen in Germany in 2015 was apparently not even linked to population development [14].

Little is known about the vector potential of Ae. koreicus. It is stated to be a vector of Japanese encephalitis virus in Russia $[1,15]$ and has proven able to transmit Dirofilaria immitis $[16,17]$ and Brugia malayi $[18]$ in the laboratory. Vector competence for chikungunya virus, although low, was also reported [19].

Aedes koreicus is closely related and both morphologically and genetically very similar to the Japanese bush mosquito Ae. japonicus. Genetic analysis, though, is believed to unambiguously lead to reliable distinction in all life stages while the species can be easily confused morphologically: major characteristics (dark subbasal band on the hind femur in Ae. japonicus but not in Ae. koreicus and a pale basal band on hind tarsomere 4 in Ae. koreicus but absent in Ae. japonicus $[9,20])$ may be inarticulate and overlooked. Taxonomically, both species belong to the Japonicus Group of the Aedes subgenus Finlaya [2]. In fact, Ae. koreicus was once considered an Ae. japonicus variant [1], and-based on genetic 
data-Cameron et al. [21] have suggested its reclassification as a subspecies of Ae. japonicus. Aedes japonicus is another invasive mosquito species originating from Asia, which was first detected in Europe in 2000 [22]. From 2003 onwards, it has spread into numerous European countries and over considerable parts of southern and northwestern Germany [23-25].

Following the submission of an Ae. koreicus female from the South German federal state of Bavaria in 2015 [9], a single larval specimen of this species was found in 2016 in a cemetery in the municipality of Wiesbaden, federal state of Hesse, western Germany, triggering further investigations in 2017 and 2018 during which local establishment of Ae. koreicus was demonstrated [20, 26, 27].

Before the background that Ae. japonicus had been found in the Wiesbaden region for the first time in 2015 and continuously and widely distributed afterwards [24, 25, 28], we thoroughly re-analysed our "Ae. japonicus" collection material from the presently known Ae. koreicus population area in Wiesbaden and several other randomly selected collection sites in Germany, similar to
Slovenian investigations in 2016 [10]. We also present $A e$. koreicus findings from 2019 and 2020 in Germany and discuss the genetic identification of this species.

\section{Methods \\ Study material}

Mosquitoes analysed included Ae. japonicus/koreicus-like specimens and were derived from larval field collections and submissions of adults to the citizen science project "Mueckenatlas" from 2019 and 2020 as well as from archived mosquito material (adults prepared from larval samplings and Mueckenatlas submissions) collected from 2015 to 2018.

Based on Vezzani et al. [29], field sampling in search of Ae. koreicus in the Wiesbaden area was conducted in cemeteries from 27 to 29 August 2019, and from 11 to 15 August and 12 to 16 September 2020, with sampling site selection according to previous Ae. koreicus detections in that area. In 2019, 12 sites were sampled, including five previously positive, five previously negative and two never sampled before (Fig. 1b, Table 1). In 2020, 17 sites were inspected, including five positive in previous

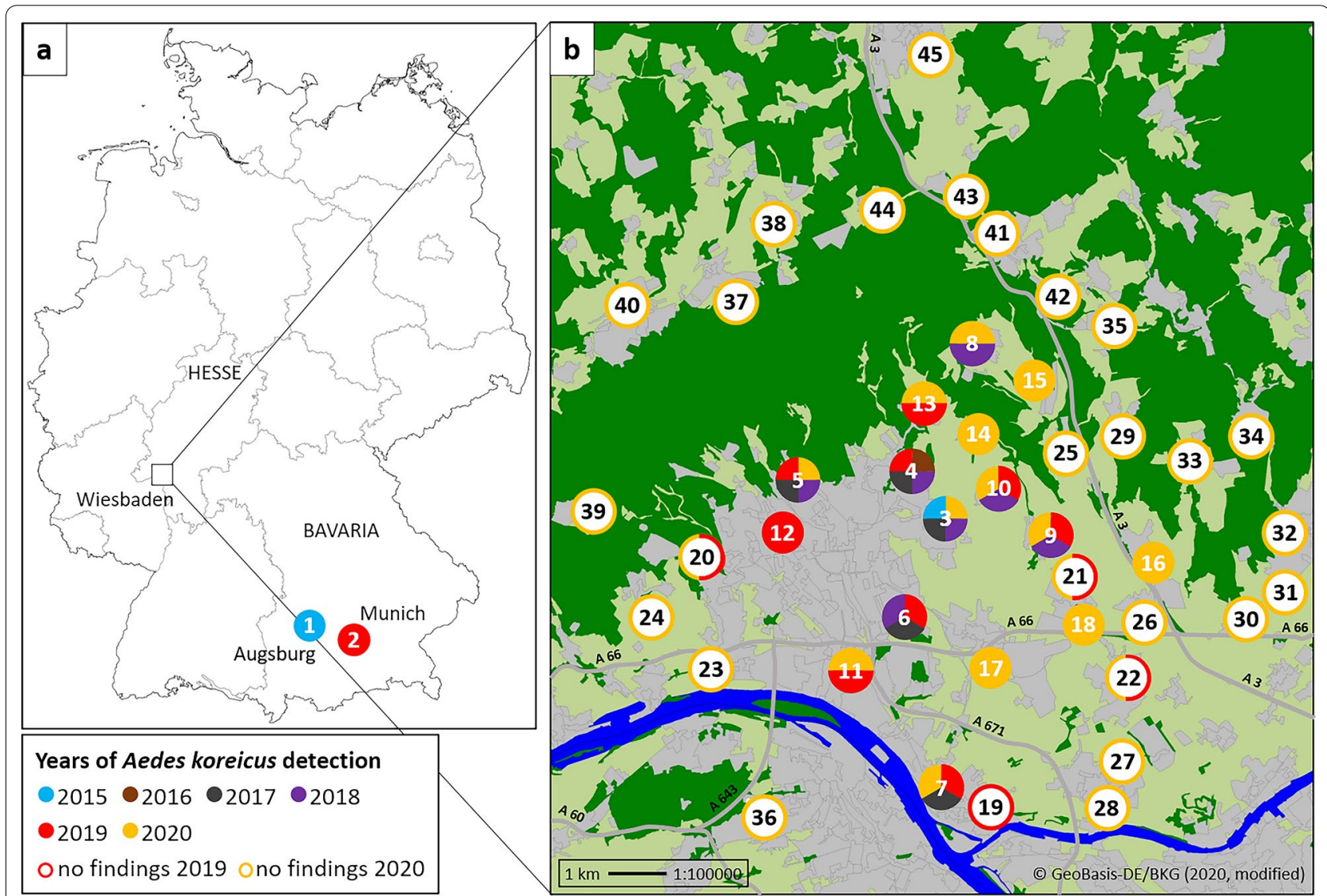

Fig. 1 Aedes koreicus collection sites in Germany, based on Werner et al. [9], Pfitzner et al. [19, 25], Steinbrink et al. [26] and the present study, as well as sampling sites found negative for Ae. koreicus in this study (see Table 1 for site details). a Overview of Germany with federal states and cities named where Ae. koreicus specimens were found. $\mathbf{b}$ Detail enlargement of the greater Wiesbaden area where the Ae. koreicus population occurs. 
Table 1 Details of sites sampled for Ae. koreicus in the municipality of Wiesbaden and the cities of Augsburg and Munich (site numbers refer to Fig. 1)

\begin{tabular}{|c|c|c|c|c|c|c|}
\hline Site No. & Location & Habitat & $\begin{array}{l}\text { Ae. } \\
\text { koreicus/Ae. } \\
\text { japonicus }\end{array}$ & Mode of collection & $\begin{array}{l}\text { Month(s)/year(s) } \\
\text { of collection }\end{array}$ & References \\
\hline 1 & Augsburg & Urban area & $+/-$ & MA submission & Jun 2015 & {$[9]$} \\
\hline 2 & Munich & Urban area & $+/-$ & 2 MA submissions & Sep 2019 & This study \\
\hline \multirow[t]{9}{*}{3} & Wiesbaden-Bierstadt & Urban area & $+/-$ & 2 MA submissions $^{\mathrm{a}}$ & Aug 2015 & This study \\
\hline & & & $+/-$ & MA submission ${ }^{\mathrm{a}}$ & Aug 2017 & This study \\
\hline & & Cemetery, field/garden & $+/-$ & Ovitrapping & Aug, Oct 2017 & {$[20,26]$} \\
\hline & & urban area & $+/$ nd & BG-Sentinel trapping & Aug-Oct 2017 & [27] \\
\hline & & & $+/$ nd & Dipping/sieving & May, July 2018 & \\
\hline & & Cemetery & $+/+$ & Dipping/sieving ${ }^{a}$ & Aug 2018 & This study \\
\hline & & & $+/-$ & & Sep 2018 & \\
\hline & & & $-/-$ & Dipping/sieving & Aug 2019 & This study \\
\hline & & & $+/-$ & & Aug 2020 & \\
\hline \multirow[t]{6}{*}{4} & Wiesbaden-Sonnenberg & Cemetery & $+/-$ & Dipping/sieving & Sep 2016 & {$[20,26]$} \\
\hline & & & $+/+$ & Ovitrapping, dipping/sieving & Aug, Oct 2017 & {$[20,26]$} \\
\hline & & & $+/-$ & Dipping/sieving & 2018 & \\
\hline & & & $+/-$ & & Aug 2019 & This study \\
\hline & & Forest & $+/-$ & Ovitrapping & Jul-Oct 2017 & {$[20]$} \\
\hline & & Industrial area & $+/-$ & & & \\
\hline \multirow[t]{4}{*}{5} & Wiesbaden-Northeast & Cemetery & $+/-$ & Dipping/sieving & Sep 2017 & {$[20,26]$} \\
\hline & & & $+/+$ & & 2018 & \\
\hline & & & $+/+$ & & Aug 2019 & This study \\
\hline & & & $+/+$ & & Aug 2020 & \\
\hline \multirow[t]{3}{*}{6} & Wiesbaden-Southeast & Cemetery & $+/+$ & Dipping/sieving & Sep 2017 & {$[20,26]$} \\
\hline & & & $+/+$ & & 2018 & \\
\hline & & & $+/+$ & & Aug 2019 & This study \\
\hline \multirow[t]{3}{*}{7} & Mainz-Kastel & Cemetery & $+/-$ & Dipping/sieving & Sep 2017 & {$[20,26]$} \\
\hline & & & $+/-$ & & Aug 2019 & This study \\
\hline & & & $+/-$ & & Aug 2020 & \\
\hline \multirow[t]{2}{*}{8} & Wiesbaden-Naurod & Cemetery & $+/-$ & Dipping/sieving & 2018 & {$[20,26]$} \\
\hline & & & $+/-$ & & Aug 2020 & This study \\
\hline \multirow[t]{3}{*}{9} & Wiesbaden-lgstadt & Cemetery & $+/+$ & Dipping/sieving & 2018 & {$[20,26]$} \\
\hline & & Rural area & $+/-$ & MA submission & Oct 2019 & This study \\
\hline & & Cemetery & $+/+$ & Dipping/sieving & Aug 2020 & \\
\hline \multirow[t]{3}{*}{10} & Wiesbaden-Kloppenheim & Rural area & $+/-$ & MA submission & Jul 2018 & This study \\
\hline & & Cemetery & $+/-$ & Dipping/sieving & Aug 2019 & \\
\hline & & Rural area & $+/-$ & 3 MA submissions & Jun 2020 & \\
\hline \multirow[t]{3}{*}{11} & Wiesbaden-Biebrich & Urban area & $+/-$ & MA submission & May 2019 & This study \\
\hline & & Cemetery & $+/-$ & Dipping/sieving & Aug 2019 & \\
\hline & & & $+/-$ & & Sep 2020 & \\
\hline 12 & Wiesbaden-Klarenthal & Urban area & $+/-$ & MA submission & Jul 2019 & This study \\
\hline \multirow[t]{2}{*}{13} & Wiesbaden-Rambach & Cemetery & $+/+$ & Dipping/sieving & Aug 2019 & This study \\
\hline & & & $+/+$ & & Aug 2020 & \\
\hline 14 & Wiesbaden-Hessloch & Cemetery & $+/-$ & Dipping/sieving & Sep 2020 & This study \\
\hline 15 & Wiesbaden-Auringen & Cemetery & $+/+$ & Dipping/sieving & Sep 2020 & This study \\
\hline 16 & Wiesbaden-Breckenheim & Cemetery & $+/-$ & Dipping/sieving & Aug 2020 & This study \\
\hline 17 & Wiesbaden-Erbenheim & Cemetery & $+/-$ & Dipping/sieving & Aug 2020 & This study \\
\hline 18 & Wiesbaden-Nordenstadt & Rural area & $+/-$ & MA submission & Oct 2020 & This study \\
\hline 19 & Mainz-Kostheim & Cemetery & $-1-$ & Dipping/sieving & Aug 2019 & This study \\
\hline
\end{tabular}


Table 1 (continued)

\begin{tabular}{|c|c|c|c|c|c|c|}
\hline Site No. & Location & Habitat & $\begin{array}{l}\text { Ae. } \\
\text { koreicus/Ae. } \\
\text { japonicus }\end{array}$ & Mode of collection & $\begin{array}{l}\text { Month(s)/year(s) } \\
\text { of collection }\end{array}$ & References \\
\hline \multirow[t]{2}{*}{20} & \multirow[t]{2}{*}{ Wiesbaden-Dotzheim } & \multirow[t]{2}{*}{ Cemetery } & \multirow[t]{2}{*}{$-1+$} & \multirow[t]{2}{*}{ Dipping/sieving } & Aug 2019 & \multirow[t]{2}{*}{ This study } \\
\hline & & & & & Aug, Sep 2020 & \\
\hline \multirow[t]{2}{*}{21} & \multirow[t]{2}{*}{ Wiesbaden-Nordenstadt } & \multirow[t]{2}{*}{ Cemetery } & \multirow[t]{2}{*}{$-/-$} & \multirow[t]{2}{*}{ Dipping/sieving } & Aug 2019 & \multirow[t]{2}{*}{ This study } \\
\hline & & & & & Aug, Sep 2020 & \\
\hline \multirow[t]{2}{*}{22} & \multirow[t]{2}{*}{ Wiesbaden-Delkenheim } & \multirow[t]{2}{*}{ Cemetery } & \multirow[t]{2}{*}{$-/-$} & \multirow[t]{2}{*}{ Dipping/sieving } & Aug 2019 & \multirow[t]{2}{*}{ This study } \\
\hline & & & & & Aug, Sep 2020 & \\
\hline 23 & Wiesbaden-Schierstein & cemetery & $-/-$ & Dipping/sieving & Aug, Sep 2020 & This study \\
\hline 24 & Wiesbaden-Frauenstein & Cemetery & $-/+$ & Dipping/sieving & Aug, Sep 2020 & This study \\
\hline 25 & Wiesbaden-Medenbach & Cemetery & $-/-$ & Dipping/sieving & Aug, Sep 2020 & This study \\
\hline 26 & Hofheim-Wallau & Cemetery & $-/-$ & Dipping/sieving & Aug, Sep 2020 & This study \\
\hline 27 & Hochheim—new cemetery & Cemetery & $-/-$ & Dipping/sieving & Aug, Sep 2020 & This study \\
\hline 28 & Hochheim_old cemetery & Cemetery & $-/+$ & Dipping/sieving & Aug, Sep 2020 & This study \\
\hline 29 & Hofheim-Wildsachsen & Cemetery & $-/+$ & Dipping/sieving & Aug, Sep 2020 & This study \\
\hline 30 & Hofheim-Diedenbergen & Cemetery & $-/-$ & Dipping/sieving & Aug, Sep 2020 & This study \\
\hline 31 & Hofheim-Marxheim & Cemetery & $-/+$ & Dipping/sieving & Aug, Sep 2020 & This study \\
\hline 32 & Hofheim & Cemetery & $-/+$ & Dipping/sieving & Aug, Sep 2020 & This study \\
\hline 33 & Hofheim-Langenhain & Cemetery & $-/-$ & Dipping/sieving & Aug, Sep 2020 & This study \\
\hline 34 & Hofheim-Lorsbach & cemetery & $-/+$ & Dipping/sieving & Aug, Sep 2020 & This study \\
\hline 35 & Eppstein-Bremthal & Cemetery & $-1-$ & Dipping/sieving & Aug, Sep 2020 & This study \\
\hline 36 & Mainz-Gonsenheim & Cemetery & $-/+$ & Dipping/sieving & Aug, Sep 2020 & This study \\
\hline 37 & Taunusstein-Wehen & Cemetery & $-1-$ & Dipping/sieving & Aug, Sep 2020 & This study \\
\hline 38 & Taunusstein-Neuhof & Cemetery & $-/-$ & Dipping/sieving & Aug, Sep 2020 & This study \\
\hline 39 & Schlangenbad-Georgenborn & Cemetery & $-/+$ & Dipping/sieving & Aug, Sep 2020 & This study \\
\hline 40 & Taunusstein-Hahn & Cemetery & $-/+$ & Dipping/sieving & Aug, Sep 2020 & This study \\
\hline 40 & Taunusstein-Bleidenstadt & Cemetery & $-/-$ & Dipping/sieving & Aug, Sep 2020 & This study \\
\hline 41 & Niedernhausen-Königshofen & Cemetery & $-1-$ & Dipping/sieving & Aug, Sep 2020 & This study \\
\hline 42 & Niedernhausen & Cemetery & $-/+$ & Dipping/sieving & Aug, Sep 2020 & This study \\
\hline 43 & Niedernhausen-Niederseelbach & Cemetery & $-1-$ & Dipping/sieving & Aug, Sep 2020 & This study \\
\hline 44 & Niedernhausen-Engenhahn & Cemetery & $-/+$ & Dipping/sieving & Aug, Sep 2020 & This study \\
\hline 45 & Idstein & Cemetery & $-1-$ & Dipping/sieving & Aug, Sep 2020 & This study \\
\hline
\end{tabular}

a Previously misidentified as Ae. japonicus; $\mathrm{MA}=$ Mueckenatlas, $\mathrm{nd}=$ no data

studies, eight previously negative, one new in this study but already checked in 2019, and three checked for the first time (Fig. 1b, Table 1). An additional 21 sites were examined outside the Wiesbaden municipality in 2020, eight of which had been inspected previously by Pfitzner et al. [20,26], while 13 were checked for the first time (Fig. 1b, Table 1).

Examined cemeteries had different sizes and structures and were located in urban and rural and close to forested areas. Potential Ae. koreicus breeding sites were generally evenly distributed over the whole cemetery areas, with all detected ones being inspected during a visit.

Samples were taken by sieving water from flower vases, flower pot saucers and water bowls, and by dipping wells. Second to fourth bush mosquito larval instars as identified on the spot with the naked eye [30] were transferred into closable glass jars filled with water from the collection site and transported to the laboratory. There they were kept in the same jars at $24{ }^{\circ} \mathrm{C}, 70 \% \mathrm{RH}$ and a $12 \mathrm{~h}$ light $/ 12 \mathrm{~h}$ darkness regime until adult emergence, with the container lid replaced by a collection net. Until pupation, larvae were fed ground fish food (Tetra TabiMin, Melle, Germany) which was carefully added onto the water of the rearing containers once per day until spreading by the surface tension came to a standstill. As soon as adult emergence started, collection nets were removed daily and exposed to overnight freezing at $-20^{\circ} \mathrm{C}$ to kill the adults while being replaced on the rearing containers. Killed adults were stored frozen or pinned at room temperature. 
In addition, Asian bush mosquitoes submitted to the Mueckenatlas in 2019 and 2020 were subjected to thorough species identification, and adult voucher specimens from our entomological collection (derived from both past Mueckenatlas submissions and field sampling), available in a dried form either in vials or pinned, were re-analysed. Particular attention was paid to archived specimens originating from the Wiesbaden area and specimens collected along a direct imaginary line from Augsburg to Wiesbaden, but randomly selected samples from other German "Ae. japonicus" distribution areas represented in the entomological collection were also checked.

\section{Species identification}

Morphological species identification concentrated on females where distinguishing characters are more prominent than in males, using the keys provided by Tanaka et al. [2] and Pfitzner et al. [20].

Females morphologically pre-identified as Ae. koreicus or with ambiguous morphological characteristics were genetically identified by sequencing of polymerases chain reaction (PCR)-amplified DNA marker regions. For this purpose, total DNA was extracted from a single leg of each mosquito using the QIAamp DNA Mini Kit (Qiagen, Hilden, Germany) according to the manufacturer's instructions. The extracted DNA was stored at $-20{ }^{\circ} \mathrm{C}$ until further processing.

PCR amplification was comparatively performed on two genetic markers: the mitochondrial ND4 (nicotinamide adenine dinucleotide dehydrogenase subunit 4) gene and the $\mathrm{CO} 1$ (cytochrome oxidase c subunit 1) gene, with four different primer pairs targeting the latter. PCR protocols and primers were used as described by Kumar et al. [31], Lehmann et al. [32] and Zielke et al. [33].

Following agarose gel electrophoresis, PCR products were extracted by means of the QIAquick Gel Extraction Kit (Qiagen) and cycled unidirectionally using the PCR primers and the BigDye Terminator v3.1 Cycle Sequencing Kit (Thermo Fisher, Darmstadt, Germany) according to the manufacturer's instructions, purified by NucleoSEQ ${ }^{\circledR}$ columns (Macherey-Nagel, Düren, Germany) and sequenced on a 3500 Genetic Analyzer (Applied Biosystems/Hitachi, Darmstadt, Germany). Selected samples intended for deposition in GenBank were sequenced bidirectionally.

The Geneious Prime programme (version 2019.2.3, Biomatters, Ltd., Auckland, New Zealand) was used for sequence editing and alignments. Genetic data were checked against GenBank (https://blast.ncbi.nlm.nih. gov/Blast.cgi) and/or BOLD databases (https://www. boldsystems.org/).

\section{Phylogenetic analyses}

ND4 sequences obtained for Ae. koreicus were compared to all corresponding sequences available in GenBank. To display genetic relationships between specimens or populations, a phylogenetic tree was calculated using the maximum likelihood method with evolutionary distances computed by the Tamura 3-parameter model [34]. Evolutionary analyses were conducted in MEGA X [35] using Ae. japonicus (KF211480) as an outgroup.

\section{Results}

\section{Mosquito field collections in 2019 and 2020}

In 2019 and 2020, cemetery collections in the Wiesbaden area produced bush mosquito larvae developing to 184 Ae. koreicus (58 in 2019, 126 in 2020) and 250 Ae. japonicus females (33 in 2019, 217 in 2020), and to 231 male specimens of either of these two Aedes species (71 in 2019, 160 in 2020). Out of 42 cemeteries sampled in the two years (Table 1), 14 were positive for Ae. koreicus, including seven of these positive for the first time, two thereof already checked by Pfitzner et al. [20, 26] (Fig. 1b). Four of the positive cemeteries were slightly outside the previously described Ae. koreicus population area $[20,26]$.

In 13 cemeteries, only Ae. japonicus was found, while in six cemeteries both Ae. koreicus and Ae. japonicus were present. East of the municipality of Wiesbaden, in the direction of Frankfurt International Airport, Ae. koreicus aquatic stages could not be found in 10 cemeteries checked in 2020 .

\section{Mueckenatlas submissions 2019 and 2020}

Six Ae. koreicus specimens were submitted to the Mueckenatlas in 2019, with four of them from three different collection sites in the Wiesbaden area and two from a single collection site in Munich (Fig. 1). In 2020, four Ae. koreicus individuals were received from two sites in the Wiesbaden area (three from one and one from a second site; Fig. 1a). The 2019 and 2020 Mueckenatlas collection sites in Wiesbaden were different, and four of the six Wiesbaden collection sites linked to Mueckenatlas submissions matched geographically with sites found positive for Ae. koreicus larvae in the field (Fig. 1b).

\section{Re-examination of archived mosquito material}

Two "Ae. japonicus" specimens collected in the Wiesbaden area in 2015 and submitted to the Mueckenatlas monitoring scheme (Fig. 1b, Table 1) turned out to be Ae. koreicus after genetic identification. In addition, two Ae. koreicus specimens submitted from that area in 2017 and 2018 (one specimen per year) were found to be misidentified as Ae. japonicus (Fig. 1a). Furthermore, a vast majority of Ae. koreicus individuals (65 of 66) were found 
in two re-analysed larval collections from the same cemetery in the Wiesbaden municipality from 2018 (Fig. 1b, Table 1).

No admixture by Ae. koreicus was found in Asian bush mosquito material collected between 2016 and 2020 from other sites around the Wiesbaden area $(n=8)$, from sites in and around Augsburg (2016-2020: 17 sites), where the first Ae. koreicus specimen ever found in Germany originated, from sites along an imaginary line from Augsburg to Wiesbaden (2016: 5 sites, 2017: 18 sites, 2018: 4 sites, 2019: 8 sites, 2020: 9 sites), or from sites arbitrarily selected throughout the German Ae. japonicus distribution area (4-8 different sites per years 2015-2018).

\section{Genetic identification}

PCR amplification of the CO1 marker region described by Hébert et al. [36] using the primers LCO1490 and HCO2198 [37] consistently produced incorrect or no amplicons from Ae. koreicus DNA. Often, gel electrophoresis revealed a shorter DNA fragment in addition to the expected one (ca. 710 base pairs [bp]) or the shorter fragment only (Fig. 2), with attempts to sequence them being unsuccessful. Alignment of the primers with two complete Ae. koreicus mitochondrial DNA sequences from GenBank (accession nos. NC_046946, MT093832) showed mismatches pertaining to two bases in the forward and four bases in the reverse primer, although not at the $3^{\prime}$-ends. PCRs with the same primers and lower

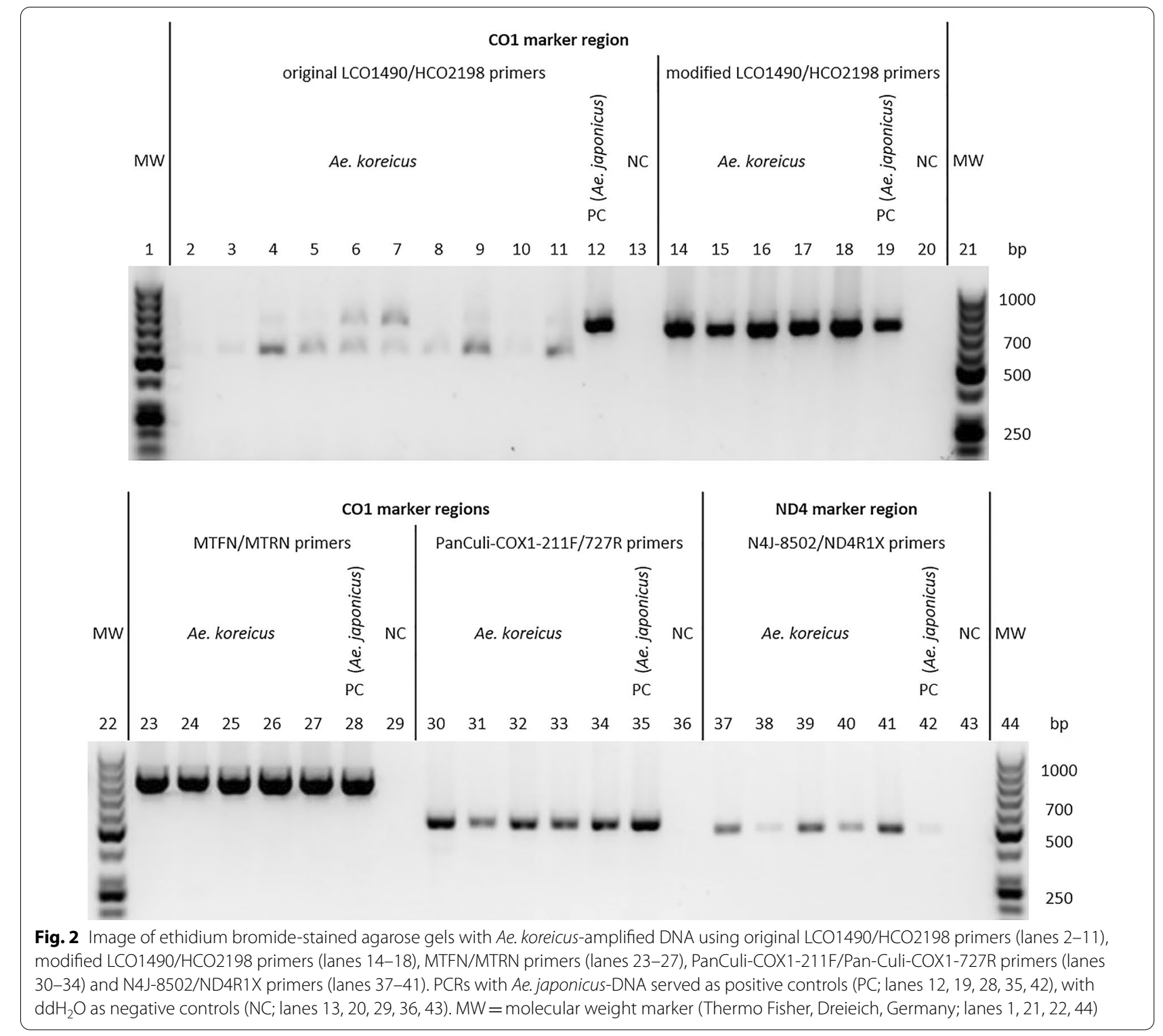


annealing temperatures $\left(50\right.$ and $52{ }^{\circ} \mathrm{C}$ instead of $\left.54{ }^{\circ} \mathrm{C}\right)$ to compensate for the base mismatches did not improve the results. Newly constructed primers LCO1490mod (5'-TCTCAACAAATCATAAAGATATTGG-3') and HCO2198mod (5'-TAAACTTCTGGGTGTCCGAAG AATCA-3'), $100 \%$ homologous to the annealing sites of the Ae. koreicus GenBank sequences, however, produced the expected PCR products (Fig. 2). These could be sequenced without difficulties, with sequences unambiguously identifying Ae. koreicus. A primer mixture consisting of equal concentrations of original and modified primers, and matching the total primer concentration according to the protocol, generated high-quality amplicons for both Ae. koreicus and 10 other arbitrarily selected Central European mosquito species (Aedes caspius, Ae.japonicus, Ae. vexans, Ae. rossicus, Anopheles claviger, Culex hortensis, Cx. territans, Cx. torrentium, Culiseta annulata, Cs. longiareolata; data not shown).

PCR amplifications using CO1-specific primer pairs according to Kumar et al. [31] (MTFN/MTRN) and Lehmann et al. [32] (PanCuli-COX1-211F/PanCuliCOX1-727R) and an ND4-specific primer pair according to Fonseca et al. [38] and Egizi and Fonseca [39] (N4J-8502/ND4R1X) also produced clear bands on the gel (Fig. 2), the corresponding DNA fragments of which could be sequenced and assigned to Ae. koreicus without problems.

\section{Phylogenetic analyses}

ND4 sequences generated from four Ae. koreicus specimens collected in this study (one each collected in Wiesbaden 2015, 2019 and 2020, one collected in Munich 2019) were deposited in GenBank under the accession numbers MZ397946-MZ397949. These were phylogenetically analysed against corresponding Ae. koreicus sequences found in GenBank: nine from Belgium, nine from Italy, five from South Korea, three from Slovenia, one from Austria and two from previous studies in Germany. The resulting phylogenetic tree, based on DNA fragments trimmed to a consistent length of $190 \mathrm{bp}$, suggests a particularly close genetic relationship of almost all specimens, with only minor differences even between individuals from Asia and Europe (Fig. 3).

\section{Discussion}

Tertiary to Ae. albopictus and Ae. japonicus, Ae. koreicus is another invasive mosquito vector species establishing populations on the European continent. After the detection of a population in the German municipality of Wiesbaden in 2016 [20, 26, 27], the present study could demonstrate that the species continued to occur in 2019 and 2020 in the delineated area where aquatic stages were identified in several cemeteries in addition to those previously found positive [20,26]. Three of the new positive sampling sites (northern and northeastern Wiesbaden) represent previously unsampled cemeteries while another four, including two previously negative (northeastern and southern Wiesbaden) and two previously unsampled ones (eastern and southeastern Wiesbaden), were located slightly beyond the borders of the population area described by Pfitzner et al. [20, 26], indicating a marginal expansion of the Ae. koreicus population after 2018. In addition, one Mueckenatlas submission each from western (Klarenthal) and southeastern Wiesbaden (Nordenstadt) (Fig. 1b) were identified as Ae. koreicus, suggesting that the species had advanced into these areas, although presence could not be confirmed by cemetery inspections. However, four out of nine Ae. koreicus Mueckenatlas submissions were received from a settlement with an Ae. koreicus cemetery record in 2019 from the northeast of the Wiesbaden municipality, close to the sites with the earliest Ae. koreicus findings in 2015 and 2016 (Fig. 1b).

No indications exist so far on where the Wiesbaden population originates from and how introduction had taken place. Since invasive mosquitoes are sometimes imported by international air traffic [40], it was hypothesised that the initial introduction might have been through Frankfurt International Airport [20], which is only some $20 \mathrm{~km}$ away. However, cemeteries of villages checked between Wiesbaden and Frankfurt International Airport did not produce any Ae. koreicus specimens. Hence, importation of larvae or eggs through ground transport, for example of gardening equipment, as seen for Ae. japonicus [14], from already colonised European areas (e.g. in Belgium or Italy) is at least similarly likely. In fact, Ae. koreicus eggs were repeatedly collected in Switzerland by oviposition traps on a service station along a major motorway connecting Italy and Germany as well as on a railway station [41], while eggs of this species were also found in an Austrian city close to a junction of major traffic axes connected to the city by various exits [11]. More in-depth population genetic studies might provide more substantiated clues as to the origin and migration routes of Ae. koreicus.

\section{Re-examination of archived mosquito samples and examination of current Mueckenatlas submissions}

After the first discovery of Ae. koreicus in Slovenia, individuals previously identified morphologically as $A e$. japonicus were re-inspected and found to be mixed with Ae. koreicus [10]. Because Ae. japonicus had also been present in Germany for years, revealing a high expansion tendency, a re-analysis of "Ae. japonicus" stock material for admixture by Ae. koreicus seemed also appropriate here. In fact, archived submissions to the citizen 


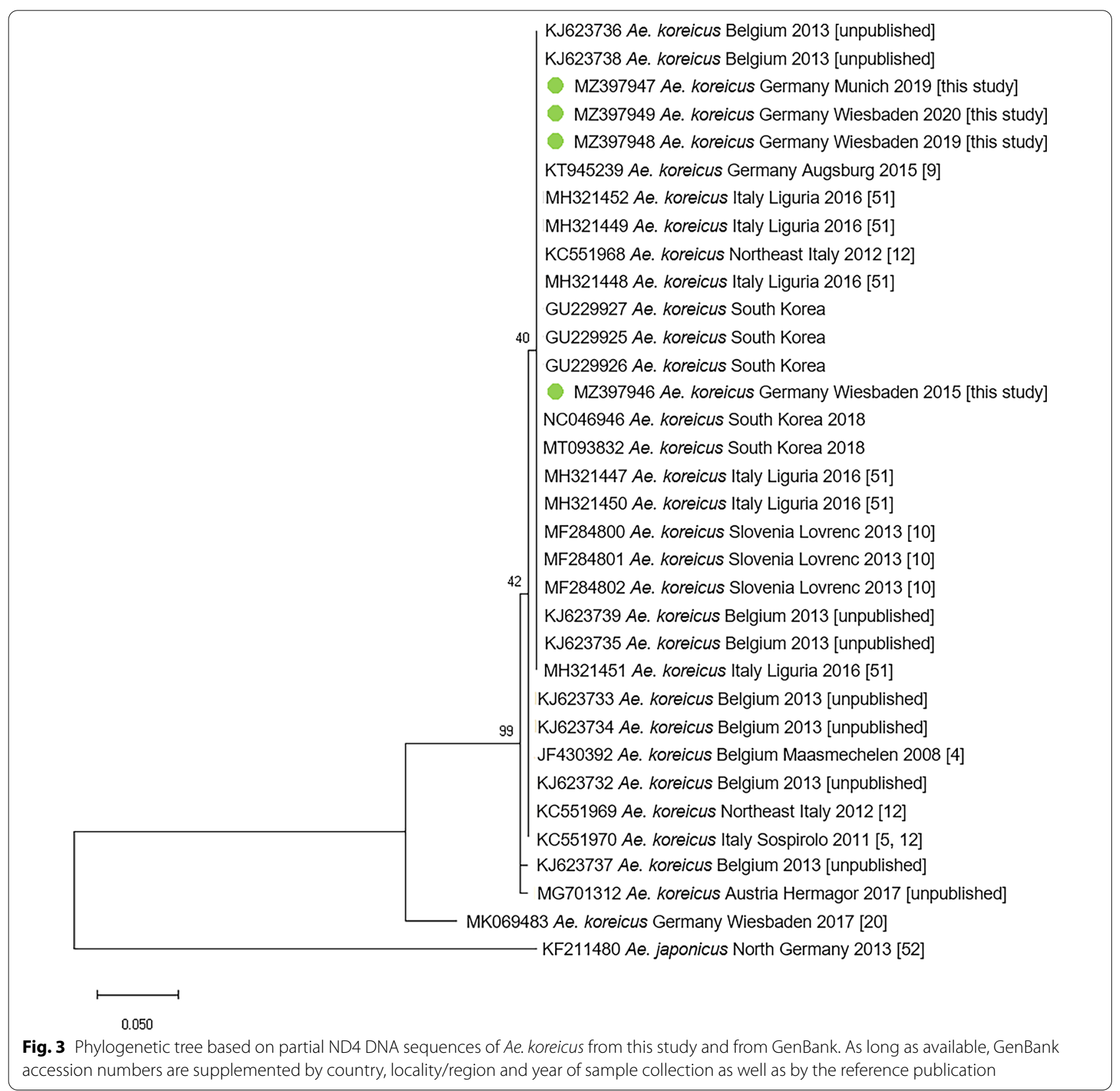

science project Mueckenatlas from the years 2015, 2017 and 2018 turned out to be Ae. koreicus by morphological and confirmatory genetic identification, suggesting that the Wiesbaden population had emerged no later than 2015. In addition, Ae. koreicus specimens were submitted during the study period 2019/2020. These findings once more demonstrate the suitability of the Mueckenatlas to detect and track invasive mosquito species $[42,43]$, but also emphasise the necessity in this global world to expect, and thoroughly check for, new alien species closely related to already established invasive ones.
Except for the Wiesbaden location, Ae. koreicus, however, could not be found among any re-inspected archived Asian bush mosquito collection material, suggesting the Wiesbaden population to be the only one in Germany. According to a field survey, also the Mueckenatlas submissions from Munich in 2019 must be considered single individual importations not connected to a resident population. 


\section{Expansion behaviour}

Even though two species as closely related and as similar in appearance as Ae. japonicus and Ae. koreicus can be found in the same area, they are not necessarily characterised by the same ecological requirements and may exhibit different behaviours. It is unclear exactly which factors are responsible for the species' ability to become established and spread. Both species originate from temperate areas in Asia [2] and can therefore be expected to be adapted to Central European climatic conditions. In fact, eggs of both species are able to overwinter in Europe, so that populations once established have remained present through the years. However, in contrast to Ae. japonicus, which generally exhibits a considerable active and passive dispersal rate in Europe, although stationary populations have been observed [24, 25], European populations of Ae. koreicus have mainly remained quite stationary and restricted to the area of initial establishment [44, 45]. Thus, the observed negligible spread of the German population fits well with studies on other European Ae. koreicus populations. The population in Belgium, which has been observed from 2008 onwards through the years, has remained restricted to an area even smaller than the German one $[44,46]$. Contrasting other European populations, however, the current Italian Ae. koreicus population has a large distribution area with noticeable spread into various parts of northern Italy up to the border with Switzerland [12]. Due to various morphological similarities, the Belgian and Italian Ae. koreicus populations are associated with an origin on Jeju-do Island, Korea $[4,5]$, where one of two Korean bush mosquito variants can be found [2]. By contrast, the German population is morphologically more similar to the population variant observed on the Korean mainland [20]. Hitherto, there has been no evidence that the Ae. koreicus variants differ in their environmental requirements or behaviour that may influence expansion dynamics. Whether the lack of expansiveness is due to ecological conditions not met in Europe or just to the specific invasive mosquito strain(s) and their genetic make-up therefore remains a question for further studies. ND4 sequence data, at least as far as the trimmed fragment available for comparison from GenBank entries is concerned, do not appear to be informative enough to deduce genetic relationships and origins, and thus to conclude on displacement and migration routes, of geographic populations.

\section{Primer evaluation}

CO1-barcoding, based on PCR with LCO1490 and HCO2198 primers according to Hébert et al. [36] and Folmer et al. [37], has been shown successful in identifying many mosquito species worldwide (e.g. [47-49]). Thus, this PCR approach has been used for routine identification of mosquitoes collected in Germany for many years [50], including attempts to genetically verify morphologically pre-identified Ae. koreicus specimens in this study. However, the above primers obviously did not anneal correctly which could be attributed to base pair mismatching with the rDNA of this specific mosquito species. By contrast, three other primer pairs, MTFN/ MTRN, PanCuli-COX1-211F/PanCuli-COX1-727R and N4J-8502/ND4R1X [31, 32, 37], targeting CO1 and ND4 genetic markers, respectively, were successfully used for molecular identification of Ae. koreicus. A slight modification of the LCO1490/HCO2198 nucleotide sequences solved the annealing problem but prevented functionality with other mosquito species, while an equal mixture of original and modified primers worked well with Ae. koreicus and other mosquito species tested. Accordingly, studies applying genetic identification of mosquitoes from an area in which Ae. koreicus occurs, but not specifically focusing on that species, should well consider which genetic marker and primer pairs to use in order to obtain unambiguous identification results and to minimise work at the same time.

\section{Conclusions}

Based on the documentation of Ae. koreicus sampling sites from 2016 to 2018 and the finding of this species at new sites in the Wiesbaden area in 2019 and 2020, it appears that the only currently known German population of Ae. koreicus is more or less stable five years after its establishment. Apparently, an expansion, if it has taken place, is negligible. However, the present evaluation was based on cemetery inspections alone, as cemeteries simultaneously offer plenty of potential mosquito breeding sites and unrestricted access to investigators [29]. Including allotment and private gardens might well show a more widespread distribution of Ae. koreicus in the Wiesbaden area than detected in this study. Spreading capacity as well as origin and introduction route(s) of Ae. koreicus are major topics that need to be addressed in more detail in the future. The demonstrated establishment and occasional introduction of this potential mosquito vector species in Germany, however, should be reason enough to continue mosquito monitoring, paying special attention to the distinction between Ae. koreicus and Ae. japonicus.

\section{Acknowledgements \\ The authors thank Susanne Fischer, Friedrich-Loeffler-Institut, Greifswald- Insel Riems, Germany, for preparing the phylogenetic tree.}

\section{Authors' contributions}

DW and HK designed the study and contributed archived and 'Mueckenatlas' mosquitoes. NH did the fieldwork, identified the collection material, analysed the data and drafted the manuscript. HK acquired funding and supervised the work. DW and HK reviewed and edited the manuscript. All authors read and approved the final manuscript. 


\section{Funding}

Open Access funding enabled and organized by Projekt DEAL.

\section{Availability of data and materials}

Data supporting the conclusions of this article are included within the article.

\section{Declarations}

\section{Ethics approval and consent to participate}

Not applicable.

\section{Consent for publication}

Not applicable.

\section{Competing interests}

The authors declare that they have no competing interests.

\section{Author details}

${ }^{1}$ Friedrich-Loeffler-Institut, Federal Research Institute for Animal Health, Insel Riems, Greifswald, Germany. ${ }^{2}$ Leibniz-Centre for Agricultural Landscape Research, Muencheberg, Germany.

Received: 23 June 2021 Accepted: 19 October 2021

Published online: 12 November 2021

\section{References}

1. Gutsevich AV, Monchadskii AS, Shtakel'berg AA. Fauna of the USSR, Diptera Vol. 3, No. 4, mosquitoes, family Culicidae. Academy of Sciences of the USSR, Zoological Institute, Leningrad, Russia; 1971 [English translation by the 'Israel Program for Scientific Translations', Keter Publishing House Ltd., Jerusalem 1974].

2. Tanaka K, Mizusawa K, Saugstad ES. A revision of the adult and larval mosquitoes of Japan (including the Ryukyu Archipelago and the Ogasawara Islands) and Korea (Diptera: Culicidae). Contrib Am Entomol Inst. 1979;16:1-987.

3. Medlock JM, Hansford KM, Versteirt V, Cull B, Kampen H, Fontenille D, et al. An entomological review of invasive mosquitoes in Europe. Bull Entomol Res. 2015;105:637-63.

4. Versteirt V, Pecor JE, Fonseca DM, Coosemans M, Van Bortel W. Confirmation of Aedes koreicus (Diptera: Culicidae) in Belgium and description of morphological differences between Korean and Belgian specimens validated by molecular identification. Zootaxa. 2012;3191:21-32.

5. Capelli G, Drago A, Martini S, Montarsi F, Soppelsa M, Delai N, et al. First report in Italy of the exotic mosquito species Aedes (Finlaya) koreicus, a potential vector of arboviruses and filariae. Parasit Vectors. 2011;4: e188.

6. Suter T, Flacio E, Fariña BF, Engeler L, Tonolla M, Müller P. First report of the invasive mosquito species Aedes koreicus in the Swiss-Italian border region. Parasit Vectors. 2015;8: e402.

7. Ganushkina LA, Patraman IV, Rezza G, Migliorini L, Litvinov SK, Sergiev VP. Detection of Aedes aegypti, Aedes albopictus, and Aedes koreicus in the Area of Sochi, Russia. Vector Borne Zoonotic Dis. 2016;16:58-60.

8. Kurucz K, Kiss V, Zana B, Schmieder V, Kepner A, Jakab F, Kemenesi G. Emergence of Aedes koreicus (Diptera: Culicidae) in an urban area, Hungary, 2016. Parasitol Res. 2016;115(12):4687-9.

9. Werner D, Zielke DE, Kampen H. First record of Aedes koreicus (Diptera: Culicidae) in Germany. Parasitol Res. 2016;115:1331-4.

10. Kalan K, Šušnjar J, Ivovic V, Buzan E. First record of Aedes koreicus (Diptera, Culicidae) in Slovenia. Parasitol Res. 2017;116:2355-8.

11. Fuehrer HP, Schoener E, Weiler S, Barogh BS, Zittra C, Walder G. Monitoring of alien mosquitoes in western Austria (Tyrol, Austria, 2018). PLoS Negl Trop Dis. 2020;14: e0008433.

12. Montarsi F, Martini S, Dal Pont M, Delai N, Ferro Milone N, Mazzucato M, et al. Distribution and habitat characterization of the recently introduced invasive mosquito Aedes koreicus [Hulecoeteomyia koreica], a new potential vector and pest in north-eastern Italy. Parasit Vectors. 2013;6:e292.

13. Gradoni F, Bertola M, Carlin S, Accordi S, Toniolo F, Visentin P, et al. Geographical data on the occurrence and spreading of invasive Aedes mosquito species in Northeast Italy. Data Brief. 2021;36:107047.
14. Kampen $H$, Schuhbauer A, Walther D. Emerging mosquito species in Germany-a synopsis after 6 years of mosquito monitoring (2011-2016). Parasitol Res. 2017;116:3253-63.

15. Miles JA. Some ecological aspects of the problem of arthropod borne animal viruses in the Western Pacific and South-East Asia regions. Bull World Health Organ. 1964;30:197-210.

16. Feng L. Experiments with Dirofilaria immitis and local species of mosquitoes in Peiping, North China, with a note on Lankesteria culicis in Ae. koreicus. Ann Trop Med Parasit. 1930;24:347-66.

17. Montarsi F, Ciocchetta S, Devine G, Ravagnan S, Mutinelli F, di Regalbono $A F$, et al. Development of Dirofilaria immitis within the mosquito Aedes (Finlaya) koreicus, a new invasive species for Europe. Parasit Vectors. 2015:8:e177.

18. KCDC. Elimination of lymphatic filariasis in Korea, National documentation for certification. National Institute of Health, Ministry of Health and Welfare, Republic of Korea. 2007.

19. Ciocchetta S, Prow NA, Darbro JM, Frentiu FD, Savino S, Montarsi F, et al. The new European invader Aedes (Finlaya) koreicus: a potential vector of chikungunya virus. Pathog Glob Health. 2018;112:107-14.

20. Pfitzner WP, Lehner A, Hoffmann D, Czajka C, Becker N. First record and morphological characterization of an established population of Aedes (Hulecoeteomyia) koreicus (Diptera: Culicidae) in Germany. Parasit Vectors. 2018;11: e662.

21. Cameron EC, Wilkerson RC, Mogi M, Miyagi I, Toma T, Kim HC, Fonseca D. Molecular phylogenetics of Aedes japonicus, a disease vector that recently invaded Western Europe, North America, and the Hawaiian islands. J Med Entomol. 2010;47:527-35

22. Schaffner F, Chouin S, Guilloteau J. First record of Ochlerotatus (Finlaya) japonicus japonicus (Theobald, 1901) in metropolitan France. J Am Mosq Control Assoc. 2003;19:1-5.

23. Kampen $\mathrm{H}$, Werner D. Out of the bush: the Asian bush mosquito Aedes japonicus japonicus (Theobald, 1901) (Diptera, Culicidae) becomes invasive. Parasit Vectors. 2014;7: e59.

24. Kampen H, Kuhlisch C, Frohlich A, Scheuch DE, Walther D. Occurrence and spread of the invasive Asian bush mosquito Aedes japonicus japonicus (Diptera: Culicidae) in West and North Germany since detection in 2012 and 2013, respectively. PLOS ONE. 2016;11: e0167948.

25. Koban MB, Kampen H, Scheuch DE, Frueh L, Kuhlisch C, Janssen N, et al. The Asian bush mosquito Aedes japonicus japonicus (Diptera: Culicidae) in Europe, 17 years after its first detection, with a focus on monitoring methods. Parasit Vectors. 2019;12:e109.

26. Pfitzner WP, Lehner A, Hoffmann D, Czajka C, Sittig N, Becker N. Current distribution of a newly established population of Aedes koreicus in southwestern Germany. Proc IXth EMCA Conf-Mosquito Control without Borders, La Rochelle, France, 11-14 March 2019; 85.

27. Steinbrink A, Zotzmann S, Cunze S, Klimpel S. Aedes koreicus-a new member of the genus Aedes establishing in Germany? Parasitol Res. 2019;118:1073-6.

28. Kampen H, Holicki CM, Ziegler U, Groschup MH, Tews BA, Werner D. West Nile virus mosquito vectors (Diptera: Culicidae) in Germany. Viruses. 2020;12: e493.

29. Vezzani D. Artificial container-breeding mosquitoes and cemeteries: a perfect match. Trop Med Int Health. 2007;12:299-313.

30. Kampen H, Zielke D, Werner D. A new focus of Aedes japonicus japonicus (Theobald, 1901) (Diptera, Culicidae) distribution in Western Germany: rapid spread or a further introduction event? Parasit Vectors. 2012;5: e284.

31. Kumar NP, Rajavel AR, Natarajan R, Jambulingam P. DNA barcodes can distinguish species of Indian mosquitoes (Diptera: Culicidae). J Med Entomol. 2007;44:1-7.

32. Lehmann K, Werner D, Hoffmann B, Kampen H. PCR identification of culicoid biting midges (Diptera, Ceratopogonidae) of the Obsoletus Complex including putative vectors of bluetongue and Schmallenberg viruses. Parasit Vectors. 2012;5:e213.

33. Zielke DE, Werner D, Schaffner F, Kampen H, Fonseca DM. Unexpected patterns of admixture in German populations of Aedes japonicus japonicus (Diptera: Culicidae) underscore the importance of human intervention. PLoS ONE. 2014;9: e99093.

34. Tamura K. Estimation of the number of nucleotide substitutions when there are strong transition-transversion and $\mathrm{G}+\mathrm{C}$-content biases. Mol Biol Evol. 1992;9:678-87. 
35. Kumar S, Stecher G, Li M, Knyaz C, Tamura K. MEGA X: molecular evolutionary genetics analysis across computing platforms. Mol Biol Evol. 2018;35:1547-9.

36. Hébert PD, Cywinska A, Ball SL, deWaard JR. Biological identifications through DNA barcodes. Proc Biol Sci Lond B. 2003;270:313-21.

37. Folmer O, Black M, Hoeh W, Lutz R, Vrijenhoek R. DNA primers for amplification of mitochondrial cytochrome c oxidase subunit I from diverse metazoan invertebrates. Mol Mar Biol Biotechnol. 1994;3:294-9.

38. Fonseca DM, Campbell S, Crans WJ, Mogi M, Miyagi I, Toma T, et al. Aedes (Finlaya) japonicus (Diptera: Culicidae), a newly recognized mosquito in the United States: analyses of genetic variation in the United States and putative source populations. J Med Entomol. 2001;38:135-46.

39. Egizi A, Fonseca DM. Ecological limits can obscure expansion history: patterns of genetic diversity in a temperate mosquito in Hawaii. Biol Invasions. 2015;17:123-32.

40. Ibanez-Justicia A, Gloria-Soria A, den Hartog W, Dik M, Jacobs F, Stroo A The first detected airline introductions of yellow fever mosquitoes (Aedes aegypti) to Europe, at Schiphol International Airport, the Netherlands. Parasit Vectors. 2017;10:e603.

41. Miyagi I, Lee KW. Morphology and biology of Aedes japonicus and Ae. koreicus observed in laboratory experiments. Jap J Sanit Zool. 1975;25:300.

42. Walther D, Kampen $\mathrm{H}$. The citizen science project 'Mueckenatlas' helps monitor the distribution and spread of invasive mosquito species in Germany. J Med Entomol. 2017;54:1790-4.

43. Pernat N, Kampen H, Jeschke JM, Werner D. Citizen science versus professional data collection: comparison of approaches to mosquito monitoring in Germany. J Appl Ecol. 2020;58:214-23.

44. Versteirt V, De Clercq EM, Fonseca DM, Pecor J, Schaffner F, Coosemans M, Van Bortel W. Bionomics of the established exotic mosquito species Aedes koreicus in Belgium, Europe. J Med Entomol. 2012;49:1226-32.
45. Kurucz K, Manica M, Delucchi L, Kemenesi G, Marini G. Dynamics and distribution of the invasive mosquito Aedes koreicus in a temperate European city. Int J Environ Res Public Health. 2020;17:e2728.

46. Deblauwe I, De Wolf K, Verle I, Schneider A, De Witte J, Vanslembrouck A, et al. Exotic mosquito species surveillance in Belgium: an overview from 2007-2018. Proc E-SOVE 21st Conf, Palermo, Italy, 22-26 Oct 2018; p 169.

47. Linton YM, Pecor JE, Porter CH, Mitchell LB, Garzón-Moreno A, Foley DH, et al. Mosquitoes of eastern Amazonian Ecuador: biodiversity, bionomics and barcodes. Mem Inst Oswaldo Cruz. 2013;108(Suppl 1):100-9.

48. Hernández-Triana LM, Brugman VA, Nikolova NI, Ruiz-Arrondo I, Barrero E, Thorne L, et al. DNA barcoding of British mosquitoes (Diptera, Culicidae) to support species identification, discovery of cryptic genetic diversity and monitoring invasive species. Zookeys. 2019;832:57-76.

49. Maekawa Y, Pemba D, Kumala J, Gowelo S, Higa Y, Futami K, et al. DNA barcoding of mosquitoes collected through a nationwide survey in 2011 and 2012 in Malawi, Southeast Africa. Acta Trop. 2021;213:e105742.

50. Werner D, Kowalczyk S, Kampen $\mathrm{H}$. Nine years of mosquito monitoring in Germany, 2011-2019, with an updated inventory of German culicid species. Parasitol Res. 2020;119:2765-74.

51. Ballardini M, Ferretti S, Chiaranz G, Pautasso A, Riina MV, Triglia G, et al. First report of the invasive mosquito Aedes koreicus (Diptera: Culicidae) and of its establishment in Liguria, northwest Italy. Parasit Vectors. 2019;12:e334.

52. Werner D, Kampen $\mathrm{H}$. The further spread of Aedes japonicus japonicus (Diptera, Culicidae) towards northern Germany. Parasitol Res. 2013;112:3665-8.

\section{Publisher's Note}

Springer Nature remains neutral with regard to jurisdictional claims in published maps and institutional affiliations.
Ready to submit your research? Choose BMC and benefit from:

- fast, convenient online submission

- thorough peer review by experienced researchers in your field

- rapid publication on acceptance

- support for research data, including large and complex data types

- gold Open Access which fosters wider collaboration and increased citations

- maximum visibility for your research: over $100 \mathrm{M}$ website views per year

At BMC, research is always in progress.

Learn more biomedcentral.com/submissions 